\title{
D2UIGP: A Development Work for the User Interface Design in Grid Portals
}

Miguel Vargas-Lombardo ${ }^{1}$, Doris Gutierrez ${ }^{2}$

${ }^{1}$ Technological University of Panama, Panama City, Panama; Research Group in e-Health and SuperComputer (Gises), Research Center for Development, Innovation of Technology Information and Communication (CIDITIC), Panama City, Panama.

Miguel.vargas@utp.ac.pa

Technological University of Panamá, Panama City, Panama.

doris.gutierrez@utp.ac.pa

\section{ABSTRACT}

Nowadays when we talk about Grid computing, it is important to emphasize the importance that it is having on the development various human areas, but it is also important to mention Grid portals and the impact these are having on the development of Grid computing, at serving as support in researches using this technology. In this paper, we present an overview of the importance of Grid portals and outstanding points will be announced on the use of $\mathrm{HCl}$ to the design of these portals, taking in consideration the usability that these must have.

\section{Indexing terms/Keywords}

Grid computing, Grid portals, $\mathrm{HCl}$, design, usability.

\section{Academic Discipline And Sub-Disciplines} Informatic;

\section{SUBJECT CLASSIFICATION}

GridComputing, $\mathrm{HCl}$

TYPE (METHOD/APPROACH)

Survey/Interview

\section{Council for Innovative Research}

Peer Review Research Publishing System

Journal: International Journal of Management \& Information Technology

Vol.4, No.3

editor@cirworld.com

www.cirworld.com, member.cirworld.com 


\section{INTRODUCTION}

Grid computing is a technology that enables coordinated exchange and use of hardware resources, software and information in virtual organizations [1]. These resources are shared through Local Area Networks (LAN), Metropolitan Area Networks (MAN), Internet and others to perform experiments and analysis of a project. [2].

The use of Grid computing resources by the scientists, through virtual organizations, looks to obtain faster and more efficiently results for their projects. Scientists from different fields (biomedicine, physics, chemistry, astronomy, environment, among others) have shown interest in Grid computing giving as result a new way of doing science, the eScience [3].

The e-Science could be defined as dynamics and globals collaboration that have been development on Internet of secondgeneration Internet. This implies on one hand, the use of technological tools to collect, integrate, share, mine, analyse and visualize large data volumes, and on the other hand, numerical simulations in the tera-scale [3].

With the increasing development and the great need on the part of scientists to obtain more resources, computing is now conceived as a service, implying the development of web interfaces, parallelization of the codes, its optimization in distributed environments and integration by through Grid portals [3]. A Grid portal is an application server that offers an online environment, insurance for collecting information on services and resources of the network [1]. From this point of view we can say that a Grid portal is a web application, which seeks to provide a more pleasant environment for its users, which is the intervention point of Human Computer Interaction $(\mathrm{HCl})$.

As a discipline, $\mathrm{HCl}$ can be defined as the responsible of the study, design and evaluation of the systems in charge of the interrelation between humans and machines to accomplish with this exchange information more efficient, with smaller errors coefficients, thus increasing the productivity of the resources used.

This article seeks to vouch the concept of Grid portals and support the creation of them using $\mathrm{HCl}$. Section II refers to Grid Portals, their characteristics and importance; Grid portals examples are present in Section III; Section IV presents recommendations for designing the Grid portals user interfaces, the conclusions are presented in section V.

\section{GRID PORTALS}

The need for collaborative science has generated demands for working environments that facilitate human communication and resource sharing among research communities [2], currently to do this we use what we know as Grid portal.

In [4], we can find a complete description about Grid portal, it says that the portals are web gateways aiming at concealing the underlying infrastructure through a pervasive, transparent, user friendly, ubiquitous and seamless access to heterogeneous and geographically spread resources.

Among the features that Grid portals should have, we can mention [2, 3, 5]:

- To have a user friendly environment.

- Allow the user to verify the performance of their research.

- Show the project results.

- Allow the Project management from any device (PDA, desktop, laptop, mobile phone) that has a web browser.

- Organize the project by research area.

- Enable to share the distributed resources of the investigation.

- Account management.

- To transfer files from different resources of the Grid.

After the previous considerations, we can deduce that the importance of Grid portals lies on provide to the final user a less complex project management, omitting the learning of multiple and complicated text commands to use the Grid; as well as eliminates the software installation process, since it only requires an available web browser [3]. In this sense we can say that the Grid portals looks to provide new community-oriented functionalities, enabling a strong collaboration, sharing and interaction among people and definitively considering people as the big resource [4].

As we saw, the Grid Portals can be used on different scientific researches areas, which has led to develop different applications like these, seeking supply the needs of each area, here are some examples of such applications.

\section{GRID PORTAL EXAMPLES}

Grid portals have been developed to meet the diverse scientific requirements; in this order can be cited:

\subsection{Genius portal grid}

In [3],[6] GENIUS is described as a portal that facilities the access, performance and monitoring of the projects on the Grid to the scientists using a web browser.

As shown in Figure 1. This model can be described as a three layer model to enhance teaching and learning of software engineering. 


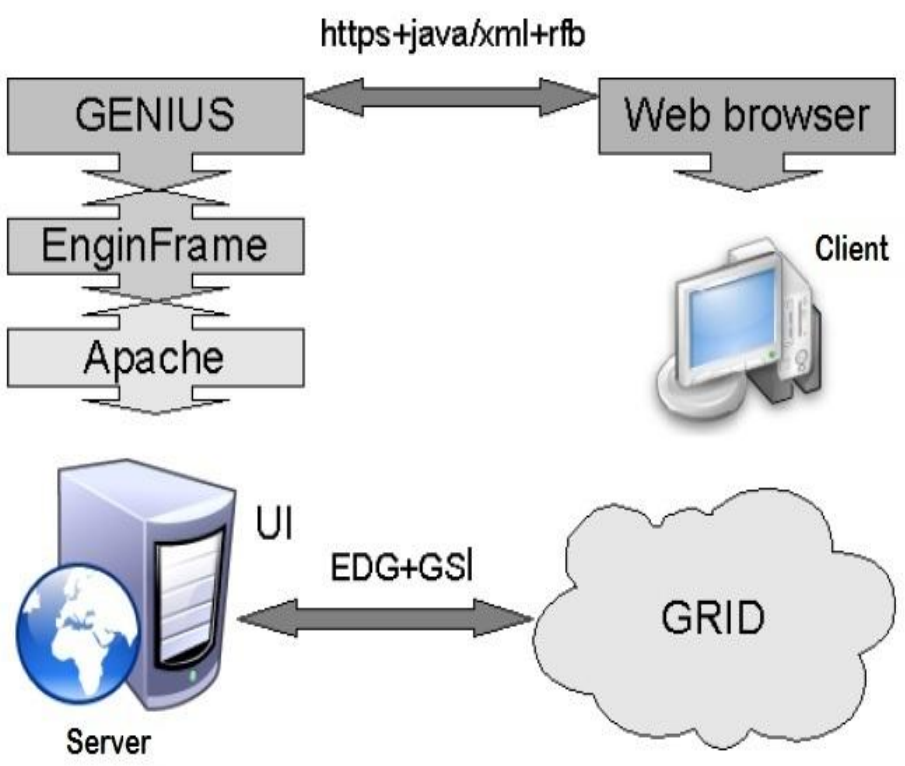

Figure 1. GENIUS Three layer model [3]

- The Client: It is the user workstation, which contains the web browser.

- The Server: Is the machine where resides the UI of gLite, concurrently with the services of Apache web, JAVA/XML, Framework Engine frame and GENIUS.

- The remote resources of the Grid.

GENIUS offers the possibility of integrating the several Web graphic interfaces applications, allowing the user to use graphical interfaces that already know to send their job to the Grid.

The main advantage of integrating Web applications with GENIUS is that generalizes the management of the Grid to all the applications instead of having to develop each of them.

\subsection{The climate-g portal}

It is a research project [4], which looks to join expertise in the field of climate change and computational science. The primary goal of Climate-G is to create a Virtual Research Environment allowing scientists and policy makers to transparently and securely perform geographical and cross-institutional data discovery, access, visualization, analysis and sharing of climate change and impacts.

The architectural design of this portal it is describe described below:

- Fabric: It represents the lowest layer, which is the physical infrastructure.

- Middleware: A set of services acting as glue. The adopted services fall in two main categories: general purpose and domain-based. The former addresses computational and data oriented general issues and needs exploiting the distributed infrastructure. The latter has to provide domain-oriented functionalities exploiting well-known, widely deployed and adopted (in the climate change community) services.

- User Interface: a Scientific Gateway represents the access point to the entire infrastructure and it provides a set of user-friendly web-based interfaces to transparently, seamlessly and securely manage collections of data and experiments, storage and computational resources, users, services, and so forth.

It is worth to mention a fundamental and orthogonal component of this architecture is security. The system has been secured by design paying special attention to authentic.

\subsection{Grid resource broker portal}

Grid Resource Broker Portal (GRB) [7] is designed to bridge the gap between those scientists scared by the complexity of the Globus Toolkit and the grid. However, GRB is much more than a user-friendly web GUI. We leverage existing functionalities in the Globus Toolkit, providing enhanced Globus services.

Among the services provided by GRB can be mentioned: user's profile management, information services, job submission, job tracking and high performance file transfers

As we saw GRB takes advantage of the Globus functionalities as a middleware, since it is the facto standard for grid computing because of its acceptance and deployment worldwide. 
The GRB services are mainly based on the following Globus services: Grid Resource Information Service (GRIS); Grid Index Information Service (GIIS); Globus Resource Allocation and Management (GRAM); Grid Security Infrastructure (GSI); GridFTP (GSI-FTP).

\subsection{Mosè}

MOSÈ [8] is a framework that supports the development, execution and management of complex geo-models. It provides a friendly environment that symbiotically combines computations, experiments, observations and geo-spatial data and provide important insights into complex phenomena. MOS`E can deliver complex grid-based solutions of geoscience problems to users wherever they have access to a web browser running on the Internet without the need to download or install specialized software or worry about setting up networks, firewall, and port policies.

The framework provides a very flexible service-oriented programming environment to design and construct large scale and computationally-intensive geoscience applications based on existing Web/Grid services technologies and standards.

A critical feature of any e-Science portal is the capability to compose workflows, to add new computational analysis programs to a catalog of workflow components and a simple way to run the workflows with the results automatically stored in the user's private data space.

MOSÈ provides a Web GUI to support easy problem set-up and data input using service classification by domaindependent ontological annotation.

We have discussed several examples of portals Grid and how these are developed to meet the needs of specific scientific area or scientific areas in general. It should be noted that the purpose of each of these portals is to facilitate the use of Grid resources, and one of the methods is emphasized to achieve this goal, using a user-friendly interface. Here are some tips to make to create applications and more efficient user interfaces.

\section{RECOMMENDATIONS FOR THE USER INTERFACE DESIGN IN GRID PORTALS}

When we talk about Grid portals, we must remember that they are Web applications, created to provide an enjoyable environment and to facilitate the final user's functions. This is why; it is appropriate to follow some rules of designing and usability, used to develop a Web application.

The following are the most important aspects that should be taken in consideration when we want to develop these kinds of applications.

\subsection{Design}

At the moment of making the design of an application, you must define the population which it will be target to. When it comes to the design of portals Grid, we known that they will be used by scientists, but also it should be taken in consideration the expertise area to which the portal will be used. This means that you should think about how information will be presented, because the way in which results are displayed to a chemical, is not the same in which they are presented to an astronomer.

After the observation made above, we conclude that each portal will have a different interface presentation, but even so, it is noteworthy that there are basic guidelines that should be applied when we are going to designing them. These guidelines include:

- Applying the principles of form (Gestalt). Perception was defined by Gestalt as a mental tendency to the order. Initially, the perception determines the entry of information, and secondly, it ensures that the resumption of the environment information allows the formation of abstractions (judgments, categories, concepts, etc.) [9]. It is necessary that the designer implements these principles into the design to facilitate the recognition of form. For example, applying the Law of Pragnanz Web page design. It is easy to fall into the trap of making a complicated design for the sake of aesthetic value or to demonstrate our ability to use the full potential that the Web offers, however, This could be tiring to the user and it will be difficult to use [10].

When we try to develop a better Grid portal user interface, we took the chance of failing at the primary goal of the same, just to want to design a flashing interface; we must to remember that the basic idea of the user interface is to presents in a more attractive way the information that the Grid offers, and that the most important thing, is to present a neatly one, that presents the information clearly and allows easy access to it.

- $\quad$ Color Psychology. The color is an effective tool for achieving usability components, in the design of Web pages. At next we mentioned some recommendations. Use color to divide the screen into different sections, making easier the detection of them. However, it should not be abused, also recalling the visual perceptual volume. Use the same background color for related areas.

- Do not overload the screen. It is important to have knowledge about color theory when you want to design. The selection of colors for the interface goes beyond the selection of pretty colors; you should keep in mind, the contrast and shades of pampering. Saturating the screen with vivid colors, will tire the user's eyes, which makes it harder to 
obtain and verify the information submitted to them. You must use a maximum of 3 to 7 colors and avoid the use of low-contrast colors such as blue-black, yellow-white.

- When you go to choose the colors to be used in a Grid portal, you should take in consideration the creation of adequate contrast for the presentation of information (background context). We recommend using complementary or contrasting colors on the color wheel and avoid using the adjacent ones [11]. Readability is better on white background and black character or a pale blue and black.

- $\quad$ Respect cultural stereotypes: red: danger, high, hot, green: forward, secure, lit. Headers and footers should be clearly differentiated from the page body this can be achieved with the use of bars or colors and borders. Use appropriate colors for the periphery and center of the retina, due to the sensitivity of the cones (blue and green background for the center).

When it comes to choose the colors to identify outstanding aspects of the interface, you must keep in mind that the meaning of these changes depends on the culture of each user; therefore you must take special care in this regard.

- Do not use background images as they interfere with good visibility and legibility.

- The title page should not exceed a maximum of $25 \%$ of it [10].

- $\quad$ The text should be justified to the left. Avoid centered paragraphs.

- Uppercase vs. Lowercase. Using both high and low in typography, especially in regard to text or content. The titles or headings can be capitalized.

- Font and size. Two or three different fonts and the same for the size. The fonts can be used to group sections of the page, apply the principles of grouping by shape, size and/or color.

- Page's Size. It is suggested that the size of each Web page does not exceed the display size or at most two, using the displacement.

- Use of icons. Use buttons with icons that indicate their functionality [12], that help the quicker understanding of the information, although it is usually helpful complement them with other encodings, such as the text label (mouse-over) [10].

Also, as any graphic design the icons must be tested to determine whether or not their represent the correct message to users. However, if already exists an icon that has been used extensively in the past, it should be used and not a new one.

Another fundamental aspect that must be taken into account when developing an application, is the degree of usability of the same, will delve into this topic and its importance.

\section{USABILITY}

When we refer to usability, we refer to the degree of acceptance that a software or hardware has with respect to the users.

In [13], it says that abstract principles offer a way of understanding usability in a more general sense, especially if we can express them within some coherent catalog. These principles are:

- Learnability: Is the ease with which new users can begin effective interaction and achieve maximal performance [13]. Table 1. Shows the categories into which the learnability is divided.

\begin{tabular}{|l|l|}
\hline Subcategories & Definition \\
\hline Searnability Subcategories
\end{tabular}

Table 1. Learnability Subcategories [13] 
In the case of Grid portals, learnability must be maximal, since scientists seeks fit very well and quickly to new functions once they begin to interact with the application, therefore introduce an environment with which they can identify, will give them more confidence and they will feel more secure with the use of the application.

- $\quad$ Flexibility: It refers to the multiplicity of way in which the user and system exchange information (Dix, Finlay et al. 2004). In this context it should be emphasized that the subcategories to mention should be taken in consideration with special attention. Table 2 . shows the categories of flexibility.

\section{Flexibility Subcategories}

\begin{tabular}{|l|l|}
\hline Subcategories & Definition \\
\hline Dialog initiative & $\begin{array}{l}\text { Allowing the user freedom from } \\
\text { artificial constraints on the input } \\
\text { dialog imposed by the system. }\end{array}$ \\
\hline Multi-threading & $\begin{array}{l}\text { Ability of the system to support } \\
\text { user interaction pertaining to more } \\
\text { than one task at a time. }\end{array}$ \\
\hline Task migratability & $\begin{array}{l}\text { The ability to pass control for the } \\
\text { execution of a given task so that it } \\
\text { becomes either internalized by the } \\
\text { user or the system or shared } \\
\text { between them. }\end{array}$ \\
\hline Substitutivity & $\begin{array}{l}\text { Allowing equivalent values of input } \\
\text { and output to be arbitrarily } \\
\text { substituted for each other. }\end{array}$ \\
\hline
\end{tabular}

Table 2. Flexibility Subcategories [13]

Dialog initiative, the user can be comfortable disabling the recommendations of the application, but there are messages that can be important in the development of the project, and should not be disabled. For the other hand the ability to multitracking, task migratability, substitutivity and customizability are important elements to consider, for his part, multi-tracking will also allow the user to verify the different phases of their project and the task migratability will improve the runtime, whether they need to a calculation with data from another task. Finally, it is the customization of the interface, which allows the user to feel more comfortable in their working environment, allowing them to do a better job.

- Robustness: Is the level of support provided to the user in determining successful achievement and assessment of goals [13].

\begin{tabular}{|l|l|}
\hline \multicolumn{2}{|c|}{ Robustness Subcategories } \\
\hline Subcategories & Definition \\
\hline Observability & $\begin{array}{l}\text { Ability of the user to evaluate the } \\
\text { internal state of the system from its } \\
\text { perceivable representation. }\end{array}$ \\
\hline Responsiveness & $\begin{array}{l}\text { Ability of the user to take } \\
\text { corrective action once an error has } \\
\text { been recognized. }\end{array}$ \\
\hline Task conformance & $\begin{array}{l}\text { How the user perceives the rate of } \\
\text { communication with the system. } \\
\text { services support all of the tasks } \\
\text { the uses wishes to perform and in } \\
\text { the way that the user understands } \\
\text { them. }\end{array}$ \\
\hline
\end{tabular}

Table 3. Robustness Subcategories [13]

In reference to Table 3, it is worth to mention the importance of the subcategories presented, since they are responsible for offer confidence to the user when interacts with the application.

The way in which the user communicates with the system is of crucial importance, so that it is willing to project status and give the ability to correct a mistake, allow to the user the opportunity to avoid waste of time. 
It is worth to mention that the above considerations only are advice, and that in any applications development process, the end-user must be taken in consideration at every stage the same.

\section{CONCLUSIONS}

Progress in Grid portal development, will coincide with the progress that can show grid computing, since they aim to serve as intermediaries between users and the resources of the Grid. Currently, there is interest from different areas for having a portal to help them facilitate their communication with Grid resources in order to concentrate on more important aspects of the research.

The development of Grid portals, covers the deeper aspects than those specified above, as are the cultural and social aspect, which also must be taken in consideration when developing the application, since they help to complement the optimal process of development.

In the application development process should be taken in consideration many aspects depending on the different point of view of the end end-user (expertise area, color use, information displays, etc.). Even though there are aspects that can be considered common in the development process, such as application design (beyond color) and the usability degree that will possess it.

\section{REFERENCES}

[1] X. Medianero-Pasco, et al., "Grid Portals: Frameworks, Middleware or Toolkit," International Journal of Computer Science Issues, 2010.

[2] Y. W. Sim, et al., "A Web/Grid Services Approach for a Virtual Research Environment Implementation," 2005.

[3] M. Oldenhof and C. Mendoza, "Integración de interfaces web con el portal grid GENIUS," 2008.

[4] S. Fiore, et al., "The Climate-G Portal: The context, key features and a multi-dimensional analysis," 2010.

[5] G. Aloisio, et al., "Grid Computing on the Web Using the Globus Toolkit," 2000.

[6] R. Barbera, et al., "The GENIUS Grid Portal, en Computing in High Energy and Nuclear Physics," 2003.

[7] G. Aloisio and M. Cafaro, "Web-based Access to the Grid Using the Grid Resource Broker Portal," 2002.

[8] G. Folino, et al., "A Grid Portal for Solving Geoscience Problems Using Distributed Knowledge Discovery Services," 2009.

[9] G. Oviedo, "La Definición del Concepto de Percepción en Psicología con Base en la Teoría de Gestalt," 2004.

[10] L. R. P. León and S. C. Vázquez, "Lineamientos ergonómicos en el diseño de páginas Web," vol. $2,2005$.

[11] L. R. Prado León and S. C. Vázquez, "Lineamientos ergonómicos en el diseño de páginas Web," Revista Ação Ergonômica, vol. 2, 2011.

[12] F. B. Tan, et al., "A Study of Web-Designers'Criteria for Effective Business-To-Costumers (B2C) Websites Using the Repertory Grid Technique," Journal of Electronic Commerce Research, vol. 10, 2009.

[13] A. Dix, et al., "Human Computer Interaction," 2004.

\section{Author's biography}

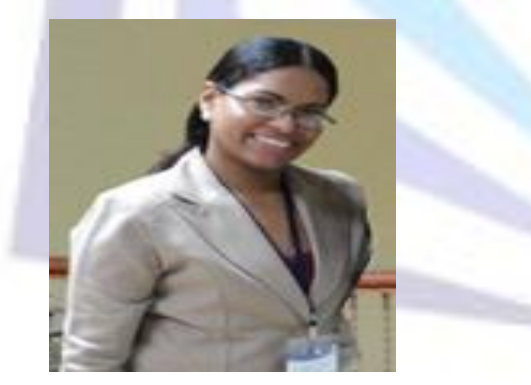

Doris Gutierrez is a student of the Master of Science in Information and Communication Technology at the Technological University of Panama. She was awarded a Bachelor's degree in Engineering and Computer Science from the Technological University of Panama in 2011. Her research interests include Human Interactive Computer.

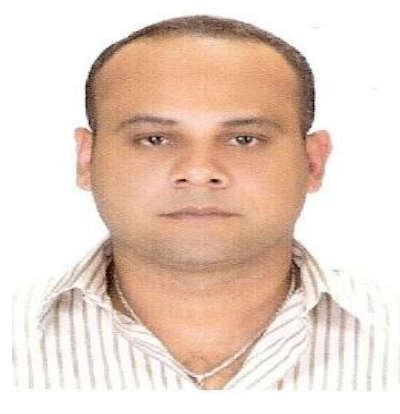

Miguel Vargas-Lombardo is a professor at the Technological University of Panama, PhD awarded by the Polytechnic University of Madrid. His main research lines are grid computing, cloud computing health-grid and e-health. 